\title{
Inteligência Artificial a Serviço da Medicina Brasileira
}

\author{
Artificial Intelligence at the Service of Brazilian Healthcare
}

\section{Gustavo Rosa Gameiroㅁ, Ana Mai Arasaki², Claudia Chi Delgado Lira ${ }^{3}$, Sadi Kneipp Neto ${ }^{4}$, Marcelo Rigotto Stachuk ${ }^{5}$}

\begin{abstract}
A Organização Mundial de Saúde acaba de lançar em seu boletim uma chamada ${ }^{1}$ para submissão de papers que mostrem aplicações da inteligência artificial (IA) no campo da saúde. Essa iniciativa busca levantar, de forma mais palpável, as principais barreiras, desafios e questões éticas relacionadas à nova tecnologia nos mais diferentes países.

Apesar de atualmente pouco explorados com a maturidade e potenciais que se esperam, os sistemas de IA vêm sendo cada vez mais utilizados a fim de contribuir para a resolução de diversos problemas de saúde e a tendência é que a participação dessa tecnologia cresça cada vez mais. Essa é uma projeção que especialistas da área concordam, como mostra o relatório Digital Health Technology Vision ${ }^{2}$ publicado em 2017 pela Accenture. Segundo tal documento ${ }^{2}$, 84\% dos executivos atuantes na área de saúde participantes da pesquisa acreditam que a inteligência artificial será responsável por importantes mudanças na forma como eles operam em alguns anos. Não é à toa que diversas instituições de saúde de referência no país como o Hospital Albert Einstein já investem nesse recurso com a criação de laboratórios com equipes de cientistas de dados dedicados a desenvolver aplicações para o cuidado de seus pacientes. Ao poder trazer soluções para muitos problemas mal resolvidos da saúde do mundo, a IA consequentemente pode gerar muito valor para todos os envolvidos seja econômico, seja social.

Mas afinal o que é Inteligência Artificial? A definição preferida do Alexandre Chiavegatto Filho, professor da Faculdade de Saúde Pública da USP na área de estatística em Saúde e Ciência de Dados, como ele nos conta no curso online de inteligência artificial ${ }^{3}$ do canal USP é: "Inteligência é a capacidade de tomar a melhor decisão possível, dada a informação disponível, com a capacidade de se adaptar a novas situações". Dessarte, a inteligência artificial pode ser classificada como tal, porque ela é capaz de dar respostas baseadas em dados disponíveis e se adaptar a novas situações. É exatamente nessa característica especial que suas diversas aplicações se baseiam, podendo auxiliar processos que humanos não conseguem executar com eficiência, sendo capas de fornecer novas possibilidades como extensão à capacidade humana.
\end{abstract}

1. Graduando do $6^{\circ}$ ano de Medicina, Faculdade de Medicina FMUSP, Universidade de São Paulo - USP. ORCID: https://orcid.org/0000-00020400-8013. Email: gustavo.gameiro@fm.usp.br.

2.Graduanda do $4^{\circ}$ ano de Design, Faculdade de Arquitetura e Urbanismo FAU, Universidade de São Paulo - USP. Email: ana.mai.arasaki@ gmail.com.

3. Graduanda do $5^{\circ}$ ano de Engenharia Elétrica, Escola Politécnica da Universidade de São Paulo - USP. Email: claudiacdlira@gmail.com. 4. Graduando do $4^{\circ}$ ano de Ciências Moleculares (Pró-Reitoria de Graduação), Universidade de São Paulo - USP. Email: sadi.neto13@gmail.com. 5. Graduando do $4^{\circ}$ ano de Ciências Moleculares (Pró-Reitoria de Graduação), Universidade de São Paulo - USP. Email: marcelorigstk@gmail.com. Endereço para correspondência: Gustavo Rosa Gameiro. Editor sênior da Revista de Medicina. Email: gustavo.gameiro@fm.usp.br. 
No campo da saúde, a tecnologia tem sido utilizada de diversas formas: na busca por diagnósticos, reconhecimento de padrões, processamentos de big data, análise de exames de imagem e auxílios à tomada de decisões perante tratamentos.

Grupos internacionais estão um passo à frente, a solução do Deep Mind, por exemplo, uma empresa britânica adquirida pelo Google, conseguiu criar um sistema capaz de diagnosticar diversas doenças a partir de exames de retinografia e até é capaz de dizer o sexo do paciente que fez o exame.

No Brasil, como um bom exemplo de aplicação dessa tecnologia, há solução do paranaense Jacson Fressato, o Robô Laura ${ }^{4}$, que executa uma tarefa que naturalmente um ser humano não seria capaz. Trata-se de um sistema inteligente que monitora continuamente os dados dos prontuários de todos os pacientes internados, a fim de detectar sinais de sepse para alertar a equipe médica com antecedência, e viabilizar um tratamento imediato para prevenir complicações e mortes evitáveis. Para operar o sistema, utilizou-se a ciência de dados e o machine learning para "ensinar" a máquina a identificar padrões nos dados dos pacientes, e discriminar aqueles que o algoritmo identifica como um paciente com sepse, e a partir disso, disparar um alerta.

De maneira similar, a Med.IA, um grupo formado por estudantes de diversos escopos da Universidade de São Paulo (USP), busca implantar uma solução de suporte à decisão utilizando a inteligência artificial no Instituto do Câncer do Estado de São Paulo (ICESP). O objetivo do projeto é ajudar os médicos a tomarem decisões relacionadas à aplicação de procedimentos que podem resultar em complicações envolvendo infecções, e aos tratamentos de antibioticoterapia para os pacientes nas UTIs do hospital. A equipe identificou um processo com bastante espaço de aprimoramento dentro do hospital: a medicação empírica de pacientes com infecção. Não existe um processo confiável de aplicação imediata de antibióticos em pacientes com infecções. A exemplo disso, há a pesquisa ${ }^{5}$ alarmante do Dr. Daniel H. Kett, publicada na revista The Lancet, que demonstrou que a aderência aos protocolos de medicação empírica para pneumonias contribuiu para o aumento da mortalidade na UTI em estudo. Pensando nessa situação de desamparo, a equipe decidiu auxiliar essa tarefa médica com um processo diferente: utilizar machine learning para ajudar médicos a terem como base informações mais confiáveis para tomarem ações criteriosas. Dessa forma, os médicos poderão ter maior certeza da adequação dos tratamentos e procedimentos que estarão aplicando em seus pacientes com infecções.

A implantação do projeto prevê a diminuição no número de ocorrências de infecções hospitalares, assim como a melhoria no tratamento delas, e consequentemente, diminuição de gastos dos hospitais com o problema e a redução dos óbitos causados por problemas envolvidos com a forma complexa e incompleta de se medicar pacientes que temos atualmente. As consequências financeiras de uma infecção são principalmente representadas pela soma do prolongamento das internações nas UTIs, estimadas em torno de $\mathrm{R} \$ 5.000,00 \mathrm{a}$ diária por paciente, e o custo com antibioticoterapia. Segundo estudos ${ }^{6}$ da Santa Casa de São Paulo, estimase que se gasta cerca de $\mathrm{R} \$ 9.400,00$ por paciente infectado, o que representa em média 2,9 vezes o custo de um paciente sem infecção. A prevenção de uma única infecção pode economizar um grande gasto. Ademais, auxiliar no tratamento correto após a contração, pode agilizar a cura, diminuindo gastos com os cuidados intensivos.

Esse tipo de projeto possui desafios comuns àqueles que utilizam inteligência artificial em hospitais, como o problema do acesso aos dados dos prontuários dos pacientes para poder aplicar a tecnologia. Como essas são informações extremamente sensíveis, faz-se necessária a aprovação de diversos setores do hospital para a concessão dos dados, mesmo que anonimizados pois o mau uso deles poderia acarretar em consequências sérias e não há ainda muita legislação sobre o tema.

Felizmente, outrossim, há maneiras confiáveis de se anonimizar dados de forma tão segura que é possível disponibilizar banco de dados de pacientes reais publicamente, como é o caso do MIMIC, criado para desenvolvimento de testes preliminares com facilidade. Foi com ele que a Med.IA conseguiu executar os primeiros testes do modelo de inteligência artificial para obter sua acurácia.

A adoção de uma cultura mais aberta à pesquisa e inovações que utilize inteligência artificial dentro do meio da saúde pode facilitar a identificação de oportunidades de melhorias em processos e operações 
do cotidiano, e o emprego de novas soluções que podem trazer benefícios muito importantes para a saúde pública. Só assim, com muita responsabilidade e ética, poderemos tirar a inteligência artificial dos Hacktons e aplicá-la de maneira significativa no cuidado do paciente, nosso objetivo maior.

\section{REFERÊNCIAS}

1.Zandi D, Reis A, Vayena E, Kenneth Goodman K. New ethical challenges of digital technologies, machine learning and artificial intelligence in public health: a call for papers [editorial]. Bull World Health Organ. 2019;97:2. doi: http:// dx.doi.org/10.2471/BLT.18.227686.

2. ACCENTURE. Artificial intelligence (AI): healthcare's new nervous system. AI: an engine for growth. Available from: https://www.accenture.com/us-en/insight-artificial-intelligence-healthcare.

3. Chiavegatto A. Curso de inteligência artificial do canal da USP. Disponível em: https://www.youtube.com/ watch? $\mathrm{v}=$ eiZoEw-_GA0\&index=3\&list=PLAudUnJeNg4tvUFZ8tXQDoAkFAASQzOHm.

4. Laura. Conheça o Robô Laura. O primeiro robô cognitivo gerenciador de risco do mundo. Disponível em: http://www. lauranetworks.com/conheca-o-robo-laura/.

5. Kett DH, Cano E, Quartin AA, Mangino JE, Zervos MJ, Peyrani P, et al. Implementation of guidelines for management of possible multidrug-resistant pneumonia in intensive care: an observational, multicentre cohort study. Lancet Infect Dis. 2011;11(3):181-9. doi: https://doi.org/10.1016/S1473-3099(10)70314-5.

6. Andrade MC. Infecção hospitalar - análise de custos. In: Jornada Norte Nordeste de Controle de Infecção Hospitalar. Disponível em: https://slideplayer.com.br/slide/285263/. 